\title{
Comparison of Endoscopic Submucosal Dissection and Surgery for Differentiated Type Early Gastric Cancer within the Expanded Criteria
}

\author{
Dong Woo Shin ${ }^{1}$, Hee Young Hwang ${ }^{2}$ and Seong Woo Jeon ${ }^{2}$ \\ ${ }^{1}$ Department of Internal Medicine, Kyungpook National University Graduate School of Medicine, Daegu, ${ }^{2}$ Department of Internal Medicine, \\ Gastric Cancer Center, Kyungpook National University Medical Center, Kyungpook National University School of Medicine, Daegu, Korea
}

Background/Aims: Endoscopic submucosal dissection (ESD) is a novel alternative treatment for differentiated early gastric cancer (EGC) without lymph node metastasis. We conducted this study to verify the therapeutic usefulness of ESD for treating differentiated EGC compared to that of surgery.

Methods: This is a retrospective cohort study of 382 patients treated with differentiated EGC from March 2006 to May 2010 . The propensity score yielded 275 matched patients. They were divided into an ESD group of 175 people and a gastrectomy group of 100 people. The patient demographics, pathologic characteristics, length of hospital stay, complication rate and survival rate were compared.

Results: The complication rate was higher for the gastrectomy group than for the ESD group ( $15.0 \%$ vs. $5.1 \%$, $p=0.007)$. The average length of patient hospitalization was longer after gastrectomy than after ESD (8.6 days vs. 2.4 days, $p<0.001$ ). There were two cases of mortality in the surgery group within 30 days of procedure. The 5-year survival rates of the two groups did not show a statistically significant difference $(92.0 \%$ vs. $93.3 \%, p=0.496)$.

Conclusions: The long-term survival rates of ESD and gastrectomy were not significantly different. The complication rate was lower for ESD than for gastrectomy, and the length of hospital stay was shorter after ESD than after gastrectomy.

Clin Endosc 2017;50:170-178

Key Words: Stomach neoplasms; Endoscopic submucosal dissection; Gastrectomy

\section{INTRODUCTION}

Early gastric cancer (EGC) is a cancer that does not invade deeper than the submucosa, irrespective of regional lymph node metastasis. ${ }^{1,2}$ Radical gastrectomy is the standard treatment method for EGC. ${ }^{3}$ However, gastrectomy has a

Received: January 4, 2016 Revised: March 31, 2016

Accepted: April 1, 2016

Correspondence: Seong Woo Jeon

Division of Gastroenterology and Hepatology, Department of Internal Medicine, Gastric Cancer Center, Kyungpook National University Medical Center, Kyungpook National University School of Medicine, 807 Hoguk-ro, Buk-gu, Daegu 41404, Korea

Tel: +82-53-200-2602, Fax: +82-53-426-8773, E-mail: sw-jeon@hanmail.net

(cc) This is an Open Access article distributed under the terms of the Creative Commons Attribution Non-Commercial License (http://creativecommons.org/ licenses/by-nc/3.0) which permits unrestricted non-commercial use, distribution, and reproduction in any medium, provided the original work is properly cited. higher mortality rate than endoscopic submucosal dissection (ESD), and it can impair the quality of life. ${ }^{4,5}$ Although laparoscopy-associated gastrectomy is less invasive than radical gastrectomy, it can result in the removal of excess normal tissue. Accumulated pathologic data of surgically resected EGC specimens has shown that most patients with EGC do not show any accompanying lymph node metastasis. Surgical gastrectomy with lymph node dissection is not always necessary for all patients, and ESD can be an excellent alternative first-line treatment for patients with a low risk of lymph node metastasis.

Nowadays, ESD and endoscopic mucosal resection (EMR) can be considered as alternative curative treatments for differentiated EGC. ESD facilitates en bloc resection of larger lesions, obtains accurate histologic diagnosis, and reduces the recurrence rate of EGC in comparison to EMR. ${ }^{6}$ According to 
the Japanese gastric cancer treatment guidelines, EMR should be performed to remove EGC with absolute indications, and ESD should be performed to remove EGC with expanded indications.?

According to the standard guidelines, the accepted indications for endoscopic resection of EGCs are: (1) differentiated elevated cancers less than $2 \mathrm{~cm}$ in diameter, and (2) non-ulcerated depressed cancers less than $1 \mathrm{~cm}$ in diameter. However, as these standard indications are strict, unnecessary gastrectomy may be performed. Consequently, several medical centers in Eastern Asia assert that these indications should be expanded. Recently, the following expanded indications for endoscopic resection were proposed: (1) differentiated mucosal cancers without ulceration, and regardless of tumor size; (2) differentiated intramucosal adenocarcinomas with ulceration, and $\leq 3 \mathrm{~cm}$ in size; (3) differentiated adenocarcinomas with minimal submucosal invasion (SM1: $\leq 500 \mu \mathrm{m}$ from the muscularis mucosae), and $\leq 3 \mathrm{~cm}$ in size; and (4) undifferentiated intramucosal cancers $\leq 2 \mathrm{~cm}$ in size. ${ }^{7.8}$ However, these criteria are restricted to investigational treatments of EGC. Since the expanded criteria were established, ESD has treated EGC in many patients. Recent studies have shown that following the standard criteria or the expanded criteria produces equivalent results in both early and long-term outcomes. ${ }^{9-11}$

In the literature, there are few studies that compare ESD with gastrectomy for EGCs within the expanded criteria. Therefore, we conducted a retrospective cohort study comparing the therapeutic results of ESD with gastrectomy to demonstrate the therapeutic usefulness of ESD for differentiated type EGCs within the expanded criteria.

\section{MATERIALS AND METHODS}

\section{Patients and methods}

This study was a retrospective cohort study and included differentiated type EGCs treated at a single tertiary medical center between March 2006 and May 2010. Patients were divided into the ESD group and the gastrectomy group according to the treatment modality. During the study, EGC patients visiting our hospital's surgery outpatient clinic were generally treated with gastrectomy; whereas, EGC patients visiting the internal medicine outpatient clinic were treated with ESD or gastrectomy according to the lesion size, presence of ulceration, and histologic type. This was not a randomized study; however, the treatment modality was generally decided randomly by the patients' choice of outpatient clinic.

For both groups, the inclusion criteria were EGCs within the expanded criteria (including the absolute criteria); treated with ESD, or gastrectomy with lymph node dissection; and differentiated type adenocarcinomas (well or moderately differentiated tubular adenocarcinoma and papillary adenocarcinoma) confirmed by pathologic examination.

The exclusion criteria were poorly cohesive carcinomas, such as signet ring cell carcinoma, confirmed by pathologic examination; EGCs recurring after previous gastrectomy or endoscopic resection; and patients lost to follow-up before 36 months without the occurrence of specific events such as death or recurrence. In the gastrectomy group, EGCs with regional lymph node metastasis confirmed by pathologic examination were also excluded from the analysis.

Small, undifferentiated EGCs without ulcerative findings could have been included in the expanded criteria for endoscopic resection, and several small studies have shown favorable outcomes from their inclusion. ${ }^{12,13}$ However, in many cases, the ulcerative findings of endoscopic examinations are obscure. In clinical practice, surgical treatment is preferentially recommended for undifferentiated EGCs. For this reason, we excluded poorly cohesive carcinomas such as signet ring cell carcinoma.

Patient demographics, pathologic characteristics, and clinical outcomes were determined by medical record review. This study was approved by the Ethics Committee of Kyungpook National University Medical Center.

\section{Pre-treatment staging}

For gastric cancer patients who were diagnosed via biopsy, contrast-enhanced abdominal-pelvic computed tomography (CT) was performed to estimate the depth of cancer invasion and detect regional lymph node metastasis or distant metastasis. In some cases, positron emission tomography (PET)CT was performed to rule out regional and distant metastasis. If there was no evidence of regional lymph node metastasis and distant metastasis, ESD was considered for treating the EGC. Prior to ESD, endoscopic ultrasonography (EUS) was performed to assess the depth of cancer invasion. When submucosal cancer invasion was identified on EUS, gastrectomy was recommended for the treatment of EGC.

\section{ESD and gastrectomy}

Two experienced endoscopists performed the ESD. For sedation, midazolam, meperidine, and propofol were injected intravenously with cardiopulmonary monitoring during the procedure. The boundary of the lesion was marked with spotty cautery by using various endoscopic knives. After injection of submucosal solutions (saline mixed with epinephrine or glycerol, and sodium hyaluronate) into the submucosal layer, a circumferential mucosal incision and dissection of the submucosal layer were performed by using an endoscopic knife. The resected ESD specimen was pinned onto a foam board, 
fixed with $10 \%$ formalin solution, and submitted to the pathologist.

Gastrectomy with extended systematic lymphadenectomy (D2 resection) was performed for the treatment of patients with EGC. In most cases, two experienced surgeons performed the gastrectomy. The surgeons decided whether to perform laparotomy or laparoscopy-assisted gastrectomy. Subtotal gastrectomy or total gastrectomy was selected according to the location of the EGC. In most patients treated with subtotal gastrectomy, Billroth I anastomosis was performed; however, a minority of patients underwent Billroth II anastomosis. In patients treated with total gastrectomy, gastrojejunal Rouxen-Y anastomosis was performed.

\section{Follow-up}

After ESD, regular periodic endoscopic examinations and contrast-enhanced abdominal-pelvic CTs were performed for the follow-up evaluation. Endoscopic examinations were scheduled at 3 and at 9 months after ESD, and annually thereafter. A pathologic examination of biopsy specimens was performed for ESD-related scars and any suspicious lesions detected during follow-up endoscopy. Contrast-enhanced abdominal CT was performed at 9 months after ESD, and annually thereafter.

For patients who underwent gastrectomy, an endoscopic examination and a contrast-enhanced abdominal-pelvic CT were performed annually to detect local EGC recurrence, regional lymph node metastasis, and distant metastasis. In some patients, a PET-CT was performed annually to detect gastric cancer recurrence.

During follow-up, an additional gastric cancer diagnosed within 1 year after ESD or gastrectomy was defined as a synchronous gastric cancer. An additional gastric cancer diagnosed at least 1 year after treatment was defined as a metachronous gastric cancer. Local recurrence was defined as a gastric cancer that recurred at the previous ESD site or gastrectomy site. We followed all patients in an outpatient office for 5 years, and recorded the date and cause of death if they passed away. We deduced the survival and mortality rate from the outpatient data.

\section{Statistical analysis}

Propensity score matching was used to reduce selection bias. The propensity score was determined by using logistic regression models. In cases where the EGC had invaded the submucosa, propensity scores were calculated. The calculated propensity scores were used to match patients in the gastrectomy group with patients in the ESD group. After propensity score matching, statistical analyses of the data were conducted with the software SPSS version 18.0 (SPSS Inc., Chicago, IL,
USA). The chi-square test was used to analyze categorical variables such as sex, comorbidities, and submucosal invasion. Numerical variables, such as age and length of hospital stay, were analyzed using an independent $t$-test. The survival rates of the two groups were compared using the Kaplan-Meier analysis and the log-rank test. A p-value of less than 0.05 was considered statistically significant.

\section{RESULTS}

\section{Characteristics of baseline demographics}

Between March 2006 and May 2010, 373 patients with EGC underwent ESD. Twenty-one patients were confirmed to have poorly cohesive adenocarcinoma or signet ring cell carcinoma by pathologic examination after ESD. One hundred thirteen patients were lost to follow-up, without the occurrence of the specific events, within 36 months after the ESD. In seven cases, the EGCs were beyond the expanded criteria. Between March 2006 and May 2010, 247 patients with EGC underwent gastrectomy with D2 lymph node dissection. Four patients had regional lymph node metastasis, and seven EGCs were confirmed after surgery to be poorly cohesive adenocarcinomas or signet ring cell carcinomas. Fifty-seven patients were lost to follow-up, without the occurrence of specific events, within 36 months after the gastrectomy, and in 29 cases the EGCs were beyond the expanded criteria. Thus, 232 differentiated type EGCs treated with ESD were compared with 150 differentiated type EGCs treated with gastrectomy. Propensity-score matching of the cohort yielded 275 matched patients. One hundred seventy-five patients were included in the ESD group and 100 patients were included in the gastrectomy group (Fig. 1).

The mean age was 61.7 years in the ESD group and 60.5 years in the gastrectomy group $(p=0.282)$. There was no statistically significant difference in the gender distribution between the two groups, with $73.7 \%$ of patients in the ESD group and $73.0 \%$ of patients in the gastrectomy group being male ( $p=0.968)$. There was no significant difference in the presence of comorbidities between the two groups, as $50.3 \%$ of patients in the ESD group and $43.0 \%$ of patients in the gastrectomy group ( $p=0.322$ ) had comorbidities. The distribution of ASA grades in the two groups was not significantly different $(p=0.534)$ (Table 1$)$.

\section{Tumor characteristics}

The proportion of the elevated type EGC was higher in the ESD group (51.4\%) than in the gastrectomy group (14.0\%, $p<0.001)$. The depressed type EGC was more common in the gastrectomy group (62.0\%) than in the ESD group (41.1\%). There was a significantly higher proportion of EGCs with 


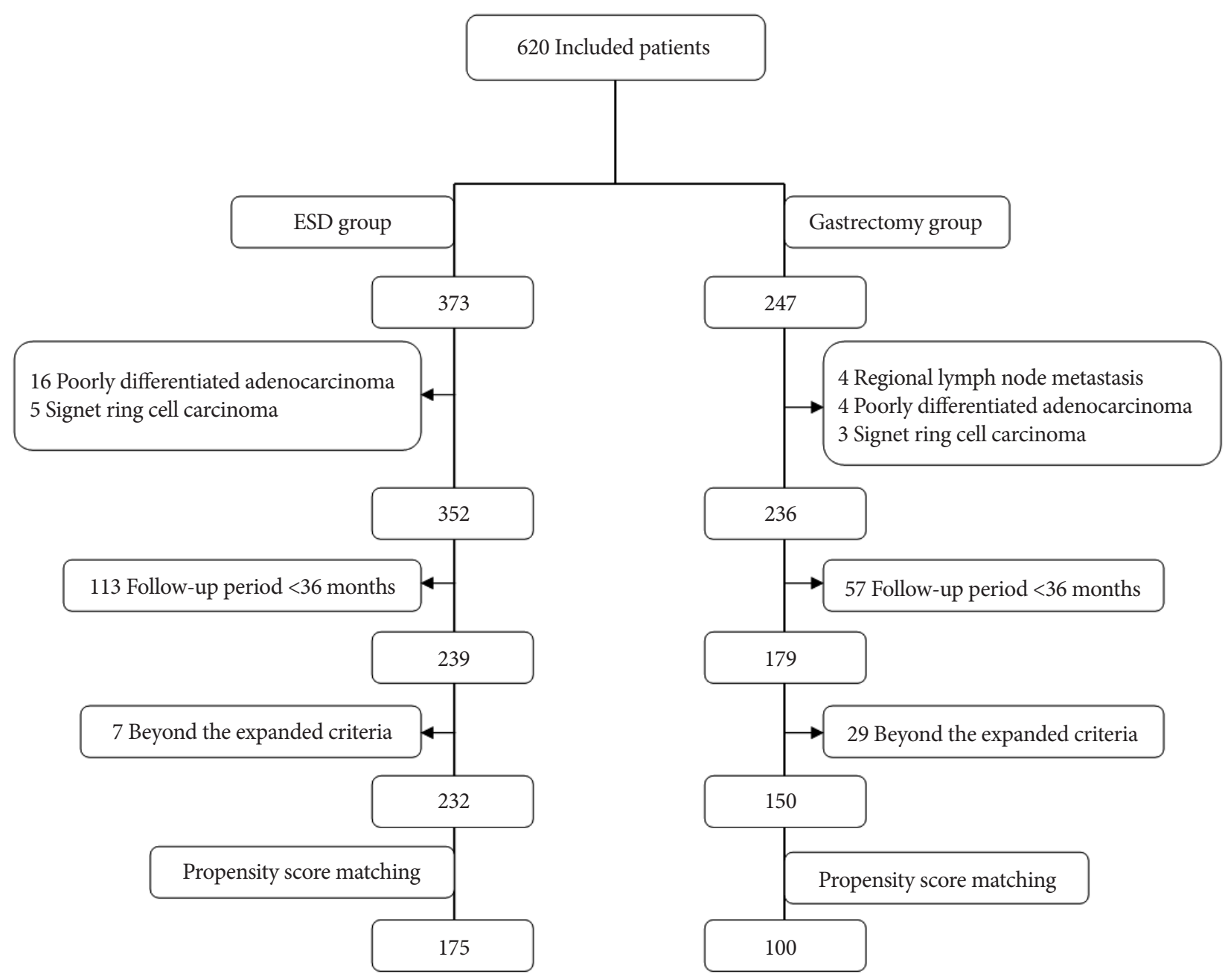

Fig. 1. Flow chart of this study.

ulceration in the gastrectomy group than in the ESD group (58.0\% vs. $16.6 \%, p<0.001)$. There was no difference in the depth of submucosal invasion between the two groups (1.7\% vs. $1.0 \%, p=0.152)$. There were no patients with a lymphatic or vascular invasion in either group (Table 1).

\section{Short-term outcomes in the ESD group and gastrec- tomy group}

Patients in the ESD group had a shorter mean hospital stay than patients in the gastrectomy group (2.4 days vs. 8.6 days, $p<0.001)$. The complication rate was lower in the ESD group than in the gastrectomy group (5.1\% vs. $15.0 \%, p=0.007)$. There was no procedure-related mortality in the ESD group, whereas two perioperative mortalities were recorded in the gastrectomy group. One patient had a cerebrovascular accident and died 10 days after gastrectomy. The other patient experienced anastomosis site leakage with intra-abdominal infection and died due to sepsis 28 days after gastrectomy.
There was one case of pathologic incomplete resection in the ESD group; however, this patient underwent subsequent gastrectomy successfully (Table 2 ).

\section{Long-term outcomes in the ESD group and gastrec- tomy group}

The median follow-up period was 56 months in the ESD group and 53 months in the gastrectomy group ( $p=0.633$ ). During the follow-up period, there was no significant difference in the risk of local recurrence or synchronous gastric cancer between the two groups. The ESD group had three cases of local recurrence (1.7\%) and six cases of synchronous gastric cancer (3.4\%). In this group, two patients with local recurrence underwent gastrectomy and the other patient, who was diagnosed with local recurrence 58 months after ESD, was successfully treated with a second ESD. After the second ESD, this patient showed no local recurrence, regional metastasis, or distant metastasis during the follow-up period. 
Table 1. Characteristics of Baseline Demographics and Tumor for the Propensity Score Matched Patients

\begin{tabular}{|c|c|c|c|}
\hline Characteristic & $\begin{array}{l}\text { ESD group } \\
(n=175)\end{array}$ & $\begin{array}{l}\text { Gastrectomy group } \\
\qquad(n=100)\end{array}$ & $p$-value \\
\hline Age, yr & $61.7 \pm 8.7$ & $60.5 \pm 9.7$ & 0.282 \\
\hline Male sex & $129(73.7)$ & $73(73.0)$ & 0.968 \\
\hline Presence of comorbidity & $88(50.3)$ & $43(43.0)$ & 0.322 \\
\hline Hypertension & $65(37.1)$ & $28(28.0)$ & \\
\hline Cardiac disease & $6(3.4)$ & $9(9.0)$ & \\
\hline Diabetes mellitus & $28(16.0)$ & $13(13.0)$ & \\
\hline CVA & $6(3.4)$ & $3(3.0)$ & \\
\hline Respiratory disease & $1(0.6)$ & $1(1.0)$ & \\
\hline Chronic renal failure & $5(2.9)$ & $2(2.0)$ & \\
\hline Chronic liver disease & $8(4.6)$ & $5(5.0)$ & \\
\hline Other malignancies & $7(4.0)$ & $4(4.0)$ & \\
\hline Others & $3(1.7)$ & $3(3.0)$ & \\
\hline \multicolumn{4}{|l|}{ ASA grade } \\
\hline 1 & $90(51.4)$ & $54(54.0)$ & 0.534 \\
\hline 2 & $61(34.9)$ & $28(28.0)$ & \\
\hline 3 & $23(13.1)$ & $18(18.0)$ & \\
\hline 4 & $1(0.6)$ & 0 & \\
\hline \multicolumn{4}{|l|}{ Lesion type } \\
\hline Elevated & $90(51.4)$ & $14(14.0)$ & $<0.001$ \\
\hline Flat & $13(7.4)$ & $24(24.0)$ & \\
\hline Depressed & $72(41.1)$ & $62(62.0)$ & \\
\hline SM invasion ${ }^{\text {a) }}$ & $3(1.7)$ & $1(1.0)$ & 0.152 \\
\hline SM1 invasion & $3(1.7)$ & $1(1.0)$ & \\
\hline Ulceration & $29(16.6)$ & $58(58.0)$ & $<0.001$ \\
\hline \multicolumn{4}{|l|}{ Lesion size } \\
\hline Major axis & $12.4 \pm 6.9$ & $15.2 \pm 8.7$ & 0.007 \\
\hline Minor axis & $8.8 \pm 4.9$ & $12.0 \pm 6.6$ & $<0.001$ \\
\hline Lymphatic invasion & 0 & 0 & 1.000 \\
\hline Vascular invasion & 0 & 0 & \\
\hline Absolute criteria & $124(70.9)$ & $71(71.0)$ & \\
\hline Expanded criteria & $51(29.1)$ & $29(29.0)$ & \\
\hline
\end{tabular}

Values are presented as mean \pm SD or number (\%).

ESD, endoscopic submucosal dissection; CVA, cerebrovascular accident; ASA, American Society of Anesthesiologists;

${ }^{a)}$ Depth of submucosal invasion (SM1: a submucosal invasion less than one-third of the depth of the submucosal layer).

Two patients with synchronous gastric cancer underwent gastrectomy and the other four patients underwent additional ESD. The gastrectomy group had one case of local recurrence $(1.0 \%)$ and one case of synchronous gastric cancer (1.0\%). In this group, the patients with local recurrence or synchronous gastric cancer were re-treated with surgery. The risk of metachronous gastric cancer was higher in the ESD group (5.1\% vs. $0 \%, p=0.029$ ). There were nine cases of metachronous gastric cancer in the ESD group. Most of these cases were successfully re-treated with ESD (four cases) or gastrectomy (four cases). One patient with metachronous gastric cancer was lost to follow-up after diagnosis, and we could not confirm the patient's course of treatment (Table 3). Survival rates were similar in the two groups. The 5-year survival rate was $92.0 \%$ in the ESD group and $93.3 \%$ in the gastrectomy group (log-rank test, $p=0.496$ ) (Fig. 2). 


\section{Cost}

We obtained the data of the summation of the individual cost elements, including the cost of the endoscopic proce-

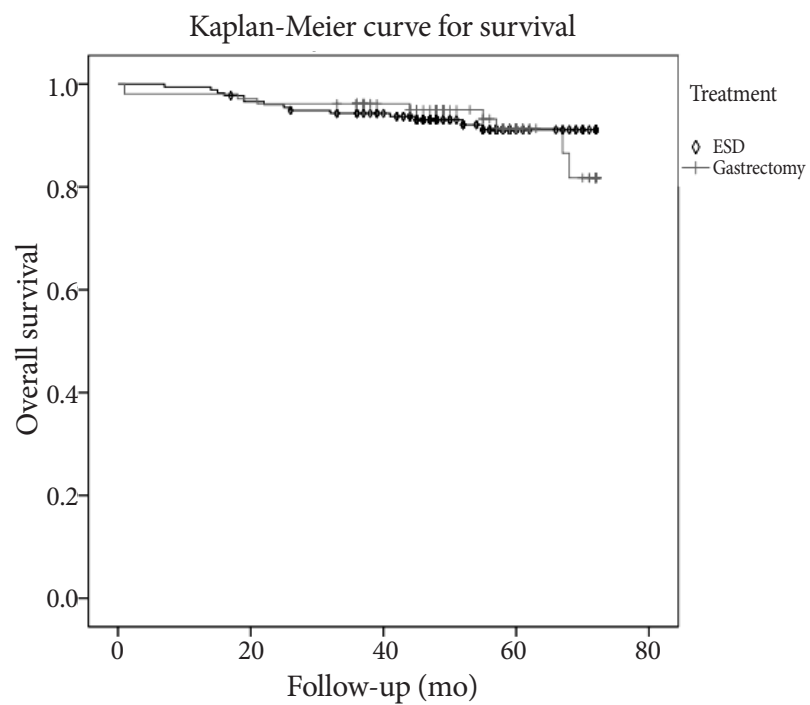

Fig. 2. Comparison of survival between endoscopic submucosal dissection (ESD) and gastrectomy group in the propensity score matched patients (log rank test, $p=0.496$ ). dure (or surgery); pathologic analysis; type of anesthesia; and length of hospital stay. We reported the average costs per patient that were calculated by the hospital charge assessment team in our center in 2014. The total cost of hospitalization for ESD was approximately $\$ 1,871$. The total costs of hospitalization for subtotal gastrectomy and total gastrectomy were approximately $\$ 5,925$ and $\$ 6,476$, respectively. ESD was less expensive than gastrectomy. These costs are based on general cases, and the total costs were higher for cases with accompanying complications after the procedure. As described in the section on short-term outcomes, complications were less common in the ESD group than in the gastrectomy group. Considering this difference, ESD seems to be more economical than gastrectomy (Table 4).

\section{DISCUSSION}

After the expanded indications were proposed, ESD was more widely used in clinical settings for the treatment of EGC. However, opinions regarding the therapeutic advantages and disadvantages of ESD compared to gastrectomy, for the ex-

Table 2. Therapeutic Outcomes of ESD and Gastrectomy Group in the Propensity Score Matched Patients

\begin{tabular}{|c|c|c|c|c|}
\hline Variable & $\begin{array}{c}\text { ESD group } \\
(n=175)\end{array}$ & $\begin{array}{l}\text { Gastrectomy group } \\
(n=100)\end{array}$ & $p$-value & OR \\
\hline Hospital stay, d & $2.4 \pm 2.1$ & $8.6 \pm 2.8$ & $<0.001$ & \\
\hline Pathologic incomplete resection, \% & $1(0.6)$ & 0 & 1.000 & \\
\hline Complication rate, $\%$ & $9(5.1)$ & $15(15.0)$ & 0.007 & $3.12(1.31-7.43)$ \\
\hline Bleeding & $4(2.3)$ & $2(2.0)$ & & \\
\hline Perforation & $4(2.3)$ & 0 & & \\
\hline Pneumonia & $1(0.6)$ & $2(2.0)$ & & \\
\hline Intraperitoneal abscess & 0 & 0 & & \\
\hline Anastomosis leak & 0 & $1(1.0)$ & & \\
\hline Adhesive intestinal obstruction & 0 & $3(3.0)$ & & \\
\hline Intra-abdominal abscess & 0 & $3(3.0)$ & & \\
\hline Atelectasis & 0 & $1(1.0)$ & & \\
\hline Pleural effusion & 0 & $1(1.0)$ & & \\
\hline Cerebrovascular accident & 0 & $2(2.0)$ & & \\
\hline 30-Day mortality & 0 & $2(2.0)$ & & \\
\hline Cerebrovascular disease & 0 & $1(1.0)$ & & \\
\hline Intra-abdominal infection & 0 & $1(1.0)$ & & \\
\hline Overall mortality & $14(8.0)$ & $12(12.0)$ & & \\
\hline Infection & $3(1.7)$ & $2(2.0)$ & & \\
\hline Other malignancy & $5(2.9)$ & $4(4.0)$ & & \\
\hline Unknown cause & $6(3.4)$ & $6(6.0)$ & & \\
\hline
\end{tabular}

Values are presented as mean \pm SD or number (\%).

ESD, endoscopic submucosal dissection; OR, odds ratio. 
Table 3. Comparison of Local Recurrence, Synchronous Gastric Cancer and Metachronous Gastric Cancer between ESD and Gastrectomy Group in the Propensity Score Matched Patients

\begin{tabular}{lccc}
\hline Variable & $\begin{array}{c}\text { ESD group } \\
(\boldsymbol{n}=\mathbf{1 7 5})\end{array}$ & $\begin{array}{c}\text { Gastrectomy group } \\
(\boldsymbol{n}=\mathbf{1 0 0})\end{array}$ & $\boldsymbol{p}$-value \\
\hline Follow-up, mo & $56(45-58)$ & $53(44-60)$ & 0.633 \\
Local recurrence, \% & $3(1.7)$ & $1(1.0)$ & 0.654 \\
Synchronous gastric cancer, \% & $6(3.4)$ & $1(1.0)$ & 0.264 \\
Metachronous gastric cancer, \% & $9(5.1)$ & 0 & 0.029 \\
5-Year survival, \% & 92.0 & 93.3 & 0.496 \\
\hline
\end{tabular}

Values are presented as median (interquartile range) or number (\%).

ESD, endoscopic submucosal dissection.

Table 4. Comparison of the Approximate Hospitalization Costs (between 2012 and 2013)

\begin{tabular}{cccc}
\hline & ESD & Subtotal gastrectomy & Total gastrectomy \\
\hline Cost & $\$ 1,871$ & $\$ 5,925$ & $\$ 6,476$ \\
\hline
\end{tabular}

ESD, endoscopic submucosal dissection.

Costs are the total cost of hospitalization for procedure or surgery. This data was about the rough total cost of hospitalization between 2012 and 2013. Costs were converted to U.S. dollars with the exchange rate of 1,050 Korean won to U.S. \$1.

panded criteria, are conflicted. In this study, we have classified the patients with EGC into the ESD group or the gastrectomy group. The patient demographics, pathologic characteristics, length of hospital stay, complication rate, and survival rate were compared. Depending on the treatment outcome, the complications, recurrence rates, and survival rates of the two groups were compared.

In this study, the duration of patient hospitalization was longer after gastrectomy than after ESD. The recovery period was longer for patients treated with gastrectomy than for patients treated with ESD. Most patients who underwent endoscopic resection were discharged a day after the procedure. There were more complications in the gastrectomy group than in the ESD group (15.0\% vs. $5.1 \%$ ). Therefore, as fewer complications, such as bleeding and perforation, were reported in the ESD group, the patients' recovery time was shorter. There were two hospital deaths $(2.0 \%)$ due to cerebrovascular accident and intra-abdominal infection in the gastrectomy group. The complication rate of gastrectomy for EGC was somewhat lower in this study than in previous studies, which reported complication rates of $24.5 \%$ and $32.5 \%{ }^{4.14}$ The complication rate (5.1\%) in the ESD group was significantly lower than the complication rate in the gastrectomy group, and it was similar to the rates reported in previous studies of $5.6 \%$ and $5.4 \% .^{10,14}$ Although there were four cases (2.3\%) of bleeding and four cases $(2.3 \%)$ of gastric perforation, there were no procedure-related mortalities in the ESD group. These patients were all treated successfully, without surgery, with an additional endoscopic procedure and conservative management.

Most of the gastric mucosa was saved in the ESD group; consequently, the prevalence rate of metachronous gastric cancers was higher in the ESD group than in the gastrectomy group. Previous studies have reported the incidence rates of metachronous gastric cancer to be $8.2 \%$ to $14 \%$ after $\mathrm{ESD}^{15,16}$ and $1.8 \%$ to $2.4 \%$ after gastrectomy. ${ }^{1718}$ In this study, the frequency of metachronous gastric cancer was much higher in the ESD group (5.1\% vs. $0 \%, p=0.029)$, although it was lower than other studies, than in the gastrectomy group. The incidence rates of synchronous gastric cancer and local recurrence were higher in the ESD group than in the gastrectomy group, but the difference was not statistically significant. The synchronous gastric cancers and metachronous gastric cancers in the ESD group were resected completely with additional ESD or surgery. Gastrectomy with lymph node dissection was recommended for the patients of recurrent gastric cancer after ESD. ${ }^{6}$ According to recent studies, local recurrence confined to the mucosa after ESD can be treated with additional ESD. ${ }^{7}$ In this study, all of the local recurrences that occurred after ESD were treated with surgery or additional ESD.

There was no statistically significant difference in the 5-year overall survival rates between the two groups. The 5-year overall survival rate was $92.0 \%$ in the ESD group and $93.3 \%$ in the gastrectomy group (log-rank test, $p=0.496$ ). In both groups, mortality was associated, not with gastric cancer, but with another disease, such as an infectious disease or another malignancy. As mentioned above, two patients died after complications from gastrectomy (cerebrovascular accident or intra-abdominal infection); however, procedure-related mortality in the ESD group was not reported. This is consistent with previous studies reporting 5 -year overall survival rates of $88 \%$ and $96.2 \%$ with ESD for EGC. ${ }^{10,19}$ A previous study of EMR versus gastrectomy for intramucosal gastric cancer showed no 
difference in survival rates between the EMR group and the gastrectomy group after the cancer was completely removed. ${ }^{20}$ Moreover, they reported a 5-year overall survival rate of $93.6 \%$ in the EMR group, which is similar to the survival rate of the EGC group in our study. However, only $71.2 \%$ of EGCs were completely removed by EMR, the remaining patients had to undergo additional management such as surgery. In our study, most patients diagnosed with EGC within the expanded criteria had the EGC completely resected by ESD. Therefore, ESD is superior to EMR in curative resection of EGC.

Although the cost of ESD and gastrectomy may differ for each country, total hospitalization costs of the ESD are much cheaper than those of gastrectomy; the total hospitalization costs of ESD are approximately one-third of the costs of gastrectomy. ESD is cheaper than gastrectomy even when additional ESD is necessary to treat synchronous or metachronous gastric cancer after endoscopic resection.

There are some limitations associated with this study. Foremost, as this is a retrospective study, the treatment chosen for gastric cancer may be associated with selection bias. However, all included cases of EGCs were within the expanded criteria, and EGCs with regional lymph node metastasis in the gastrectomy group were excluded from the analysis. Furthermore, we analyzed differentiated type EGCs, and excluded poorly cohesive carcinomas such as signet ring cell carcinomas. If our study had included poorly differentiated EGCs the results may have been somewhat different, because poorly differentiated EGCs are frequently associated with lymph node metastasis; therefore, ESD should be carefully considered when determining the type of treatment for EGCs. Second, the endoscopic treatment did not completely exclude the possibility that surgical treatment may be required later. As there is the rare possibility of lymph node metastasis in patients meeting the expanded criteria, careful surveillance with all the available modalities to detect metachronous gastric cancers is needed. It is possible to predict the probability of lymph node metastasis on the basis of the histopathologic results (i.e., the depth of invasion, degree of differentiation, vascular involvement, and lymphatic involvement). If a submucosal invasion or a lymph node metastasis is suspected, conventional surgery should be recommended. Finally, a relatively small number of EGCs were included in this study. To confirm our findings, randomized, prospective, long-term follow-up studies are needed. It is almost impossible to conduct randomized and prospective studies in a clinical situation; however, large-scale multicenter retrospective studies and cohort reviews are now being released. The expanded indication group from a recent large-scale multicenter retrospective study showed excellent short-term and long-term post-ESD outcomes without the need for additional treatment with gastrectomy. ${ }^{21}$ The compli- cation rates in the ESD and gastrectomy group were $8.5 \%$ and $28.2 \%$, respectively. The duration of hospital stay was significantly shorter in the ESD group than the gastrectomy group. ${ }^{22}$ These results have demonstrated the possibility that ESD will be an effective, first-line treatment for EGC in the future.

In conclusion, our study demonstrates that ESD for EGC treatment is associated with improved short-term outcomes, including a shorter hospital stay, lower complication rate, and lower total hospitalization cost, when compared with gastrectomy. By preserving the stomach, ESD may also improve the quality of life. There was no significant difference in the 5-year overall survival rate of the ESD and the gastrectomy. ESD could be an effective first-line curative management for differentiated-type EGCs when combined with proper patient selection and a regular follow-up program.

\section{Conflicts of Interest}

The authors have no financial conflicts of interest.

\section{REFERENCES}

1. Sano T, Kobori O, Muto T. Lymph node metastasis from early gastric cancer: endoscopic resection of tumour. Br J Surg 1992;79:241-244.

2. Carter KJ, Schaffer HA, Ritchie WP Jr. Early gastric cancer. Ann Surg 1984;199:604-609.

3. Okamura T, Tsujitani S, Korenaga D, et al. Lymphadenectomy for cure in patients with early gastric cancer and lymph node metastasis. Am J Surg 1988;155:476-480.

4. Kodera Y, Sasako M, Yamamoto S, et al. Identification of risk factors for the development of complications following extended and superextended lymphadenectomies for gastric cancer. Br J Surg 2005;92:1103-1109.

5. Folli S, Morgagni P, Roviello F, et al. Risk factors for lymph node metastases and their prognostic significance in early gastric cancer (EGC) for the Italian research group for gastric cancer (IRGGC). Jpn J Clin Oncol 2001;31:495-499.

6. Gotoda T. Endoscopic resection of early gastric cancer. Gastric Cancer 2007;10:1-11.

7. Japanese Gastric Cancer Association. Japanese gastric cancer treatment guidelines 2010 (ver. 3). Gastric Cancer 2011;14:113-123.

8. Gotoda T, Yanagisawa A, Sasako M, et al. Incidence of lymph node metastasis from early gastric cancer: estimation with a large number of cases at two large centers. Gastric Cancer 2000;3:219-225.

9. Oda I, Oyama T, Abe S, et al. Preliminary results of multicenter questionnaire study on long-term outcomes of curative endoscopic submucosal dissection for early gastric cancer. Dig Endosc 2014;26:214-219.

10. Choi MK, Kim GH, Park do Y, et al. Long-term outcomes of endoscopic submucosal dissection for early gastric cancer: a single-center experience. Surg Endosc 2013;27:4250-4258.

11. Ohnita K, Isomoto H, Shikuwa S, et al. Early and long-term outcomes of endoscopic submucosal dissection for early gastric cancer in a large patient series. Exp Ther Med 2014;7:594-598.

12. Abe S, Oda I, Suzuki H, et al. Short- and long-term outcomes of endoscopic submucosal dissection for undifferentiated early gastric cancer. Endoscopy 2013;45:703-707.

13. Kim YY, Jeon SW, Kim J, et al. Endoscopic submucosal dissection for early gastric cancer with undifferentiated histology: could we extend the criteria beyond? Surg Endosc 2013;27:4656-4662.

14. Chiu PW, Teoh AY, To KF, et al. Endoscopic submucosal dissection (ESD) 
compared with gastrectomy for treatment of early gastric neoplasia: a retrospective cohort study. Surg Endosc 2012;26:3584-3591.

15. Takeda J, Toyonaga A, Koufuji K, et al. Early gastric cancer in the remnant stomach. Hepatogastroenterology 1998;45:1907-1911.

16. Hosokawa O, Kaizaki Y, Watanabe K, et al. Endoscopic surveillance for gastric remnant cancer after early cancer surgery. Endoscopy 2002;34:469-473.

17. Nakajima T, Oda I, Gotoda T, et al. Metachronous gastric cancers after endoscopic resection: how effective is annual endoscopic surveillance? Gastric Cancer 2006;9:93-98.

18. Nasu J, Doi T, Endo H, Nishina T, Hirasaki S, Hyodo I. Characteristics of metachronous multiple early gastric cancers after endoscopic mucosal resection. Endoscopy 2005;37:990-993.

19. Goto O, Fujishiro M, Kodashima S, Ono S, Omata M. Outcomes of endo- scopic submucosal dissection for early gastric cancer with special reference to validation for curability criteria. Endoscopy 2009;41:118-122.

20. Choi KS, Jung HY, Choi KD, et al. EMR versus gastrectomy for intramucosal gastric cancer: comparison of long-term outcomes. Gastrointest Endosc 2011;73:942-948.

21. Nakamura K, Honda K, Akahoshi K, et al. Suitability of the expanded indication criteria for the treatment of early gastric cancer by endoscopic submucosal dissection: Japanese multicenter large-scale retrospective analysis of short- and long-term outcomes. Scand J Gastroenterol 2015;50:413-422.

22. Kim DY, Hong SJ, Cho GS, et al. Long-term efficacy of endoscopic submucosal dissection compared with surgery for early gastric cancer: a retrospective cohort study. Gut Liver 2014;8:519-525. 